\title{
Los mam de México y Guatemala: un pueblo binacional entre la autonomía y la heteronomía
}

\section{The Mam of Mexico and Guatemala: A Binational People between Autonomy and Heteronomy}

\author{
Miguel Ángel Cristhian Toledo Pineda \\ (1D) https://orcid.org/0000-0001-7030-6775 \\ El Colegio de la Frontera Sur \\ matoledo@ecosur.edu.mx \\ Enrique Coraza de los Santos \\ (D) https://orcid.org/0000-0003-2572-7516 \\ El Colegio de la Frontera Sur \\ ecoraza@ecosur.mx
}

\section{Resumen:}

En el presente artículo se realiza un acercamiento a los discursos y las prácticas de dos organizaciones del pueblo maya mam ubicadas en la región transfronteriza entre México y Guatemala. Por un lado está la asociación civil Conciencia Cultural Mam, en los municipios de Unión Juárez y Cacahoatán, Chiapas, y por el otro, el Consejo del Pueblo Mam de Sibinal, en el municipio de Sibinal, departamento de San Marcos. Se elabora una aproximación analítica considerando el surgimiento de las organizaciones, así como los objetivos que persiguen, desde las categorías de autonomía y heteronomía indígenas.

Palabras clave: identidad indígena, cultura indígena, autoridad ancestral, territorio, pueblo transfronterizo.

\section{Abstract:}

This article approaches the discourse and practices of two organizations of the Maya Mam people located in the Mexico-Guatemala transborder region. On the one hand, there is the non-profit civic association called Conciencia Cultural Mam (Mam Cultural Awareness) in the municipalities of Unión Juárez and Cacahoatán, Chiapas State, Mexico, and, on the other, the Consejo del Pueblo Mam de Sibinal (the Sibinal Mam People Council) in the municipality of Sibinal, Department of San Marcos, Guatemala. The analytic approach that was developed considers the emergence of these two organizations, as well as the goals they pursue, using the categories of indigenous autonomy and heteronomy.

Keywords: indigenous identity, indigenous culture, ancestral authority, territory, transborder peoples. 


\section{Introducción}

ste texto es resultado de la investigación y el acompañamiento con dos organizaciones del pueblo maya mam de reciente creación, pero con una interesante historia de lucha contra la desaparición de su cultura y de los modos de vida correspondientes a sus raíces ancestrales: por un lado, el Consejo del Pueblo Mam del municipio de Sibinal, departamento de San Marcos, Guatemala, y por el otro, la Asociación Civil Conciencia Cultural Mam, en los municipios de Unión Juárez y Cacahoatán, estado de Chiapas, México.

El propósito es indagar en los discursos y las prácticas de ambas organizaciones para conocer, en primer lugar, si existe en ellas una noción de autonomía y, en segundo, para contribuir a las reflexiones en torno a las categorías de autonomía y heteronomía considerando la importancia de estos conceptos en las luchas de los pueblos indígenas.

En efecto, existen diversos estudios que asignan relevancia a las luchas por la autonomía expresada desde los pueblos y las comunidades indígenas (López, 2006; González et al., 2010; López y Rivas, 2004; Velasco, 2003; Rivera, 2005). Para López y Rivas, la autonomía es

un proceso de resistencia mediante el cual, las etnias o pueblos soterrados, negados u olvidados fortalecen o recuperan su identidad a través de la reivindicación de su cultura, derechos y estructuras político-administrativas. En forma genérica, la autonomía, esto es, regirse uno mismo por sus leyes, es definida como la capacidad de individuos, gobiernos, nacionalidades, pueblos y otras entidades y sujetos de asumir sus intereses y acciones mediante normativas y poderes propios, opuestos en consecuencia a toda dependencia o subordinación heterónoma (López y Rivas en García y Lopez, 2016:141).

Rivera (2005) sostiene que la autonomía adopta distintos matices, alcances y relaciones con el Estado en cuestión, y por ello no se agota en una clasificación cerrada o limitada. En ese sentido, la construcción de autonomía protagonizada por los pueblos indígenas plantea la existencia de la diversidad del concepto, así como de una gran pluralidad de actores que la enarbolan y practican en distintos grados y con sus respectivas particularidades.

En contraste, la heteronomía es entendida por Franco (2017) como un régimen opuesto al de autonomía, donde las colectividades atribuyen un origen divino o natural a las leyes y normas que los gobiernan. En el caso de los pueblos indígenas, la heteronomía se asocia con una relativa dependencia con respecto a las 
políticas gubernamentales, cuyo diseño no responde a las necesidades particulares en la toma de decisiones de la organización comunitaria, y deja de lado las identidades culturales que la componen y las diversas figuras de su conformación (Hernández et al., 2004).

Considerada por Monterroso (2010) como una de las poblaciones más antiguas del área que comprende la Sierra Madre de Chiapas y la Sierra de los Cuchumatanes en Guatemala, la población mam ha tenido un largo proceso de reconfiguraciones territoriales y políticas. El sometimiento y la explotación de la fuerza de trabajo indígena mam se acentuó con la conquista y la colonización española por medio de mecanismos como la encomienda, los repartimientos o el despojo de las tierras agrícolas. Posteriormente, durante los procesos de independencia de la corona española y la adopción del sistema finquero, las clases dominantes y los dueños de las fincas dieron continuidad a la dependencia económica, política y a la sobreexplotación de la fuerza de trabajo de la población indígena mam (Quintana y Rosales, 2006; Monterroso, 2010). Durante los años setenta del siglo XIX, y antes del auge de la producción cafetalera mediante el sistema de fincas, la región del Soconusco se caracterizaba por su extremado aislamiento y escasa población, donde unas dos mil familias mam vivían de la producción agrícola para el autoconsumo (Bartra, 1995).

Durante el siglo XIX, distintos episodios históricos explican que el pueblo mam y sus manifestaciones culturales quedaran finalmente divididos entre México y Guatemala, en un contexto de conflictos territoriales entre ambos países (Spenser, 1988). El primer momento fue la incorporación de la provincia de Chiapas a México en 1824 y la separación del Soconusco de Chiapas en ese mismo año; el segundo, la reincorporación del distrito del Soconusco a Chiapas en 1842; y por último, la firma del tratado de límites entre México y Guatemala en 1882, de lo cual resultó que Chiapas y el Soconusco quedaran adheridos al territorio mexicano (De la Torre, 2015). De ese modo, los mam que quedaron en el lado mexicano fueron naturalizados como mexicanos y sus tierras se declararon propiedad de la nación (Robles, 2017). A su vez, aquellas tierras del Soconusco a las que se etiquetó como propiedad de la nación mexicana, durante el gobierno porfirista fueron ofrecidas a colonos extranjeros, en nombre del progreso nacional, y los indígenas se convirtieron en fuerza de trabajo sometida a condiciones de explotación intensa (Tarrío y Concheiro, 2006).

La Revolución mexicana tuvo repercusiones en Chiapas y la región del Soconusco hacia 1914, con el arribo de un representante de las fuerzas carrancistas al poder 
Ejecutivo estatal: el general Jesús Castro. Posteriormente, en 1921, siendo gobernador del estado el general Tiburcio Fernández, se promulgó la Ley Agraria. Sin embargo, durante el periodo posrevolucionario distintos factores impidieron un reparto agrario efectivo -falta de voluntad política, ausencia de un fuerte movimiento campesino y límites de la legislación federal y estatal- (Reyes y López, 1994). En la gestión presidencial de Lázaro Cárdenas, en la segunda mitad de la década de 1930, el inicio del reparto agrario privilegió la región del Soconusco e incluyó como beneficiarios a los peones acasillados -conformados por trabajadores indígenas mam, entre otros- de la zona cafetalera (Reyes, 2002). Fue este el contexto de conformación de los ejidos aledaños a las fincas cafetaleras en Unión Juárez y Cacahoatán, en la zona sierra que comprende la región del Soconusco.

Actualmente, en la región transfronteriza ${ }^{1}$ que comprende parte del Soconusco en México y del departamento de San Marcos en Guatemala, existen habitantes de los ejidos, aldeas, cantones y caseríos que se asumen como descendientes mayas mam en las faldas del volcán Tacaná (Tac'nan o 'nuestra madre' en lengua mam), ${ }^{2}$ que se organizan con base en el rescate de su raíz cultural y en los conocimientos asociados al legado de sus ancestros.

En la siguiente imagen (figura 1) se aprecia la ubicación del Consejo del Pueblo Mam del municipio de Sibinal, Guatemala, y de la Asociación Civil Conciencia Cultural Mam, en Unión Juárez y Cacahoatán, México.

\section{Desarrollo de argumentos y resultados}

\section{Acercamiento al universo de estudio}

Actualmente, los mam se ubican en las regiones Soconusco, Sierra y algunas comunidades dispersas en la selva del estado de Chiapas en México; y en los departamentos de San Marcos, Huehuetenango, Quetzaltenango y Retalhuleu en el altiplano de Guatemala (Quintana y Rosales, 2006). De enero a junio de 2017 se llevó a cabo una investigación cualitativa en parte del territorio señalado y se

\footnotetext{
1 Se entiende por región transfronteriza «un espacio de vida que traspasa las líneas de separación (límite) y origina una integración entre los territorios colindantes. Este espacio surge frente a las asimetrías generadas por el capital, el Estado y la transnacionalización que dan origen a un conjunto de prácticas sociales que tienen a la frontera como referencia» (Tapia, 2017:68).

2 Es importante considerar que en México existen por lo menos cuatro variantes de la lengua mam referidas por Medina (1973): una corresponde a la región Sierra, en los municipios de La Grandeza, El Porvenir, Bellavista y Siltepec; otra hablada en Cacahoatán; una tercera en Tuxtla Chico; y una última variante en Mazapa de Madero y Amatenango de la Frontera.
} 
Figura 1. Ubicación del Consejo del Pueblo Mam de Sibinal, Guatemala y la Asociación Civil Conciencia Cultural Mam en Unión Juárez y Cacahoatán, México.

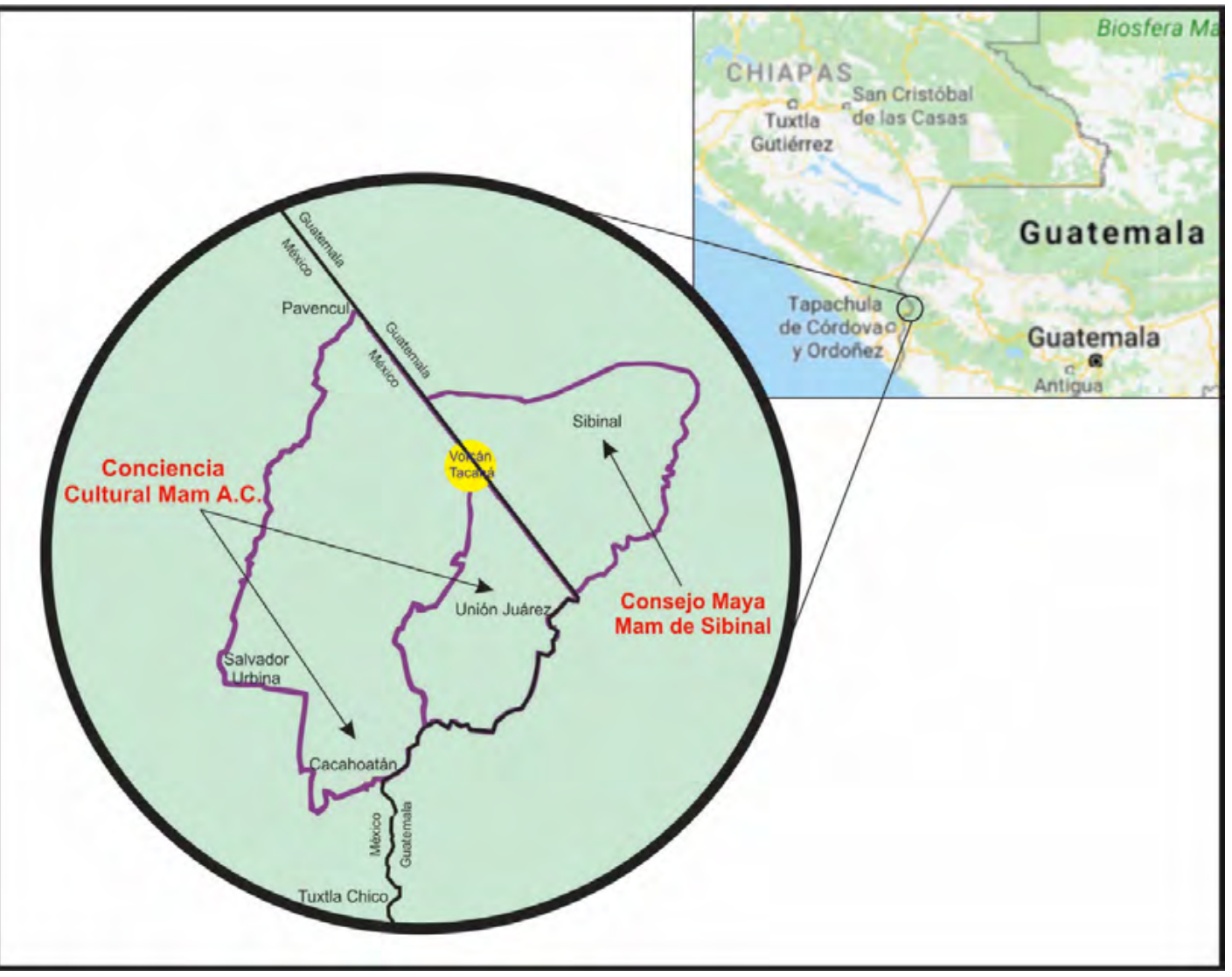

Fuente: Elaboración propia con base en la delimitación geográfica proporcionada por google maps.

realizó un acercamiento con diferentes consejos, grupos y organizaciones que se asumen como integrantes del pueblo mam en México y Guatemala.

Se recorrieron los municipios de San Marcos, San Pedro Sacatepéquez y Sibinal, pertenecientes al departamento de San Marcos en Guatemala, donde se estableció contacto con tres organizaciones principales: el Consejo del Pueblo Maya, el Consejo del Pueblo de Sibinal y el Consejo Comunitario de Desarrollo de la Aldea Chocabj. Por su parte, en México se realizaron distintas visitas en los municipios de Unión Juárez, Cacahoatán y Tapachula, donde se encuentran la Asociación Civil Conciencia Cultural Mam, la organización Raíces de Nuestra Cultura Mam y el Consejo Regional Indígena Mam del Soconusco.

Como resultado de estos acercamientos se realizó un recorte de observación con dos organizaciones y se seleccionó al Consejo del Pueblo Mam de Sibinal y a 
la Asociación Civil Conciencia Cultural Mam como sujetos de estudio para conocer si existe una noción de autonomía en sus discursos y prácticas.

La selección de ambos colectivos se basó en sus características. Se observaron similitudes y diferencias en torno a sus actividades tendientes o no a la construcción de autonomías, lo cual ha permitido un estudio comparativo. Lo anterior, siguiendo la idea de Sartori y Morlino de que «las comparaciones que sensatamente nos interesan se llevan a cabo entre entidades que poseen atributos en parte compartidos (similares) y en parte no compartidos (y declarados no comparables)»(Sartori y Morlino, 1999:35). Además, se retoma la definición de Colino (2009) de método comparado, considerado como un procedimiento científico-lógico para analizar comparativamente la realidad social entre dos unidades macrosociales para llegar a la comprobación de una hipótesis o a la interpretación de pautas causales en cada caso.

La elección de las organizaciones partió del contacto con la diversidad de los grupos mam; se eligieron solo dos debido, en primer lugar, a que son organizaciones constituidas formalmente y con las cuales era viable dar seguimiento a sus actividades y conocer sus discursos; en segundo lugar, por su cercanía geográfica, puesto que ambas se encuentran en los límites fronterizos de México y Guatemala, y en consecuencia podrían observarse con claridad los elementos de similitud en el desenvolvimiento de las prácticas asociadas a la cultura mam, ya que su proximidad posibilita los vínculos identitarios.

Por medio de la invitación de los $\operatorname{tatas}^{3}$ o abuelos con conocimiento cultural ${ }^{4}$ -pertenecientes a la asociación Raíces de Nuestra Cultura Mam- a una de las asambleas generales de la asociación civil Conciencia Cultural Mam, a mediados de 2016, se pudo entrar en contacto con la junta directiva y, posteriormente, asistimos a algunas de sus reuniones y actividades. Con respecto al Consejo del Pueblo Mam de Sibinal, durante una estancia de trabajo de campo fuimos instados por autoridades comunitarias de la aldea Chocabj, del municipio de Sibinal, a la celebración de su primer aniversario, en febrero de 2017. Ahí se estableció

3 Tata es una expresión utilizada por los mam para designar a los padres o abuelos que forman parte de su pueblo y con este adjetivo se expresa respeto y admiración hacia la persona en cuestión, a la que también se le atribuye autoridad moral. Aunque, según el diccionario náhuatl de Veracruz (Jerónimo et al., 2007), la raíz del término tata proviene del náhuatl tahtli, entre los indígenas mam es un vocablo de uso cotidiano.

4 Se entiende para este análisis la categoría de conocimiento cultural como un conjunto de «conocimientos construidos históricamente por los pueblos, por lo general en sus espacios, habitados como territorios ancestrales con los que están vinculados y de los que dependen, y que se expresan en una forma específica de existencia, en su modo particular de habitar y vivir en su mundo» (Limón, 2010: 19). 
un primer diálogo con los tatas e integrantes del consejo, a quienes después se visitó en distintas ocasiones para conocer sus actividades como autoridades ancestrales.

Como resultado de estos acercamientos se identificaron de inicio los siguientes elementos de similitud entre las dos organizaciones: a) evidencian la necesidad de un rescate de la cultura mam en su discurso y en sus actividades; b) ponen de manifiesto el reconocimiento por parte de las comunidades a las figuras de autoridad moral maya mam, es decir, a los tatas o abuelos, quienes a su vez son integrantes activos en ambos grupos; c) están integradas por un número semejante de miembros (27 y 29), mayoritariamente del género masculino y de edad superior a los 40 años; d) geográficamente se ubican en municipios con límites fronterizos binacionales entre México y Guatemala.

Como elementos de diferencia: a) se ubican en distintos países; b) en cuanto a las figuras de organización y sustentos jurídicos, en un caso es un consejo y en el otro es una asociación civil; c) no sostienen relaciones políticas similares ni con organizaciones indígenas ni con el Estado, las instituciones y los gobiernos locales oficiales; d) no coinciden en sus motivaciones, e) ni sus objetivos, prácticas y logros se encaminan en la misma dirección.

Por otra parte, nos acercamos a las organizaciones mam desde la estrategia metodológica del sujeto (Sandoval, 2016), reconociendo una postura ética y política a favor de la construcción de autonomías, pero respetando en todo momento sus procesos organizativos. Por ello recurrimos a la técnica de la observación participante. Esta aproximación significó un intento por comprender los contextos particulares en los que se estas se desenvuelven, así como las potencialidades y limitaciones que pueden presentarse con respecto a sus eventuales aspiraciones autonómicas.

Consideramos al pueblo mam de México y de Guatemala como integrante de los procesos de resistencia indígenas desarrollados desde la conquista española en América Latina (Jiménez, 2008; Smeke, 2000; Gatica, 2015). Nos basamos en afirmaciones de los indígenas organizados con los que tuvimos contacto, quienes reivindicaron su raíz cultural más allá de las condiciones adversas a las que fueron sometidos durante los procesos de conquista, colonización y asimilación republicana, en una tensión constante entre sus propias formas de vida y las que les fueron impuestas. De ese modo, observamos a las organizaciones mam desde su subjetividad política (Bonvillani, 2014), categoría que permite comprender los procesos organizativos de los sujetos de estudio visualizando la tensión que 
existe entre su desenvolvimiento y los poderes instituidos. La subjetividad permite pensar a los sujetos como condensación de prácticas, imaginarios y aspiraciones que cambian constantemente y detectar las capacidades latentes que pueden activarse desde sus realidades particulares (Alonso y Sandoval, 2015). Por lo anterior, realizamos un proceso reflexivo para reconocer el potencial organizativo de los sujetos de estudio, lo cual se explica en las siguientes líneas.

Al iniciar el trabajo de campo en el departamento de San Marcos, durante la primera fase de la observación participante y el acompañamiento con los grupos mam, se sostuvo la idea de que existía una aspiración autonómica por parte de los indígenas organizados como Consejos del Pueblo. Esta idea se generó a raíz del acercamiento a algunos integrantes del Consejo del Pueblo Maya y su proyecto político, el cual se ha difundido por medio del texto El tiempo de los pueblos, ${ }^{5}$ publicado en diciembre de 2014. En este documento exigen, como pueblos indígenas, la construcción de un Estado plurinacional en Guatemala que «respete y ejerza la democracia desde abajo, desde nuestra participación y desde nuestros territorios» (CPO, 2014: 56). Los integrantes del Consejo del Pueblo Maya proponen la construcción y constitución de los Gobiernos Autónomos de los Pueblos Indígenas con una visión de futuro, como una aspiración de organización a nivel comunitario, municipal y departamental.

Por lo que respecta a México, el levantamiento indígena del Ejército Zapatista de Liberación Nacional de 1994 puede ubicarse como un referente que ha influido de manera importante en las reivindicaciones de autonomía de los pueblos indígenas latinoamericanos (Bengoa, 2000). Rosalva Aída Hernández (2001) ha documentado la participación de los indígenas mam de la región Sierra en el llamado del movimiento zapatista hacia la creación de regiones autónomas pluriétnicas y que se concretó en 1997 con la anexión de los municipios de Motozintla, Bellavista, Amatenango de la Frontera y Frontera Comalapa al recién creado municipio autónomo de Tierra y Libertad. Asimismo, Peña y Fábregas (2015) ubican en el 2000 la creación del Comité Pro Creación del Municipio Autónomo del ejido de Pavencul, en el municipio de Tapachula, como inicio de un proceso dirigido a la conformación de un municipio libre, autónomo y soberano. Ante ello, el gobierno estatal enfrentó la situación destinando apoyos que mejoraron la infraestructura y urbanización del ejido.

5 El nombre completo del documento es: "El tiempo de los pueblos. Proyecto político. Un nuevo Estado para Guatemala: democracia plurinacional y Gobiernos Autónomos de los Pueblos Indígenas". 
No abordaremos aquí el tema del seguimiento a los procesos organizativos promovidos en la región Sierra. La información disponible no nos ha permitido ubicar una relación con las áreas de estudio ni con los grupos mam seleccionados. En consecuencia, partimos del acercamiento de grupos mam de los municipios de Unión Juárez y Cacahoatán a grupos del departamento de San Marcos, en Guatemala, que derivó en la realización del Primer Encuentro de Sabidurías Ancestrales para la Defensa de la Vida, la Madre Tierra y sus Bienes Naturales, en febrero de 2016, en la ciudad fronteriza de Tapachula, organizado por iniciativa de las autoridades mam del municipio de San Marcos, Guatemala, pertenecientes al Consejo Nacional de Autoridades Ancestrales y a la organización Liga Maya Internacional, con la intención de propiciar un «reencuentro de los pueblos, del diálogo, la convivencia y armonía, para construir lazos de hermandad y establecer procesos de ejercicio y respeto de nuestros derechos, de nuestra Madre Tierra y sus bienes naturales» (Declaración de pueblos originarios, 2016). En la declaración final del encuentro se lee una exigencia hacia los Estados nacionales y las empresas extranjeras: «Exigimos el respeto de nuestros derechos de autonomía y libre determinación como pueblos originarios frente a los modelos de 'desarrollo' que vulnera los derechos individuales y colectivos de los pueblos originarios» (Declaración de pueblos originarios, 2016: 2).

A partir de estas consideraciones (en las que por un lado hay una propuesta organizativa sólida, al menos en el discurso, de construcción de autonomía en Guatemala y, por el otro, la búsqueda de una vinculación de al menos dos grupos mam de México con organizaciones mayas del país vecino) planteamos como pregunta de investigación si existe una noción de autonomía en el Consejo del Pueblo Maya Mam de Sibinal en Guatemala y en la Asociación Civil Conciencia Cultural Mam en México y, de ser así, cuáles son las características de cada una.

Con base en dicho método asumimos el Consejo como caso paradigmático para demostrar, siguiendo estrategias de similitud, si existe una marcada diferencia con respecto al de la Asociación Civil y así establecer explicaciones causales (Pérez, 2008).

\section{Consejo del Pueblo Maya Mam de Sibinal}

En la década de los sesenta del siglo XX, la represión sistemática instrumentada por el Estado de Guatemala contra la población indígena, y que se agudizó du- 
rante los años ochenta en el contexto del conflicto armado interno, puso freno a las demandas y exigencias sociales y políticas de los pueblos y las comunidades mam. Los principales reclamos durante este periodo fueron los de participación, justicia y dignidad de los pueblos mayas a través de organizaciones como el Movimiento Indio Nacionalista, el Movimiento Revolucionario Indígena o el Movimiento Indio Tojil (González et al., 2010: 322 ). Posteriormente, estas demandas fueron expresadas a partir de la firma de los Acuerdos de Paz en 1996 y con la consolidación del movimiento maya y de las nuevas organizaciones mayas o mayanistas en la década de los noventa ( De León y Bastos, 2015).

Con la apertura del país a las actividades extractivistas, a principios del siglo $X X I$, se ha generado una articulación social de las comunidades indígenas que «se oponen a estas actividades en primer lugar porque ponen en riesgo la precaria economía comunitaria, y que no van a dejar ningún beneficio concreto a cambio» ( De León y Bastos, 2015: 61 ). En el departamento de San Marcos se registra el resurgimiento de los consejos de autoridades ancestrales, ${ }^{6}$ en el marco de la lucha del pueblo mam de Sipakapa contra los nocivos efectos ambientales generados por la mina Marlin en 2005. A partir de las movilizaciones de los mam en Sipakapa se inició un proceso de fortalecimiento o conformación de los consejos de autoridades ancestrales que más adelante se articuló en el Consejo del Pueblo de Occidente en 2008, integrado por los consejos respectivos de los pueblos Maya-Mam, Maya-Sipakapense, Maya-Popti, Maya-K'iche' y Maya-Kaqchikel.

Dicha forma organizativa y su relevancia en la toma de decisiones de las comunidades mayas tiene antecedentes previos a la conquista española, ya que en el sistema político indígena estuvo presente un consejo del pueblo donde los principales (ancianos) participaban en las decisiones trascendentales de las comunidades (Lenkersdorf, 2016). La autoridad de los tatas o abuelos entraña una perspectiva ética en la conformación de los consejos mam, en cuyas diná-

6 De León afirma que las autoridades ancestrales o indígenas son «la expresión organizada de las comunidades indígenas que, bajo los valores de la cosmovisión maya y la aplicación de los principios de legitimidad y representatividad, ordenan, adecuan y orientan el comportamiento de la sociedad comunitaria con la finalidad de favorecer y establecer el bienestar colectivo. La autoridad indígena es la persona o personas que prestan un servicio a la comunidad y al pueblo por la misma experiencia de vida y conocimientos que han adquirido, deben contar con determinadas virtudes que siempre están asociadas a su fecha de nacimiento y nawal» (De León Ardiano, 2016: 11).

7 Durante el gobierno de Óscar Berger se ratifica en 2005 el Central American Free Trade Agreement (CAFTA) en Guatemala. En ese mismo año la compañía Montana Exploradora de Guatemala S.A., que pertenece a la empresa canadiense Goldcorp, comenzó las operaciones del proyecto minero Marlin Proyect (Jiménez, 2008). 
micas sociales y políticas existe una reivindicación por el conocimiento cultural (Limón, 2010) que articula y comunica a las generaciones de jóvenes y que, de alguna manera, simboliza la memoria colectiva en el presente como recurso para proyectar el futuro.

En la actualidad, en Guatemala, la figura de consejo es un referente de organización a nivel comunitario, municipal y departamental, pero ello es resultado de la recuperación de una modalidad de autogobierno de los pueblos anterior a la Colonia, por lo que es posible pensarla como una expresión de resistencia. Así lo afirma Eliú Orozco, integrante del Consejo del Pueblo Maya, quien además recuerda que uno de los logros del pueblo maya posteriores a la firma de los Acuerdos de Paz en 1996 fue la legalización de los Consejos de Desarrollo. ${ }^{8}$ Afirma que se le llamó así «evocando a los consejos comunitarios que el pueblo maya ha traído como la figura de conducción política, económica y social en las comunidades y sobre todo en el pueblo maya; es por eso que la asamblea comunitaria es la máxima autoridad del Cocode (Consejo Comunitario de Desarrollo)» (E. Orozco, comunicación personal, 5 de agosto de 2017).

Hablando específicamente del Consejo del Pueblo Mam de Sibinal, tata Lencho, uno de los abuelos con mayor autoridad moral, al hablar del objetivo del consejo narra cómo surgió la idea de organizarse a partir de su contacto con el Consejo Nacional de Autoridades Maya Mamª y el Consejo Mam de San Marcos Txe Chman:

Y entonces ya viene la idea que cuál era el plan del consejo de los pueblos ¿ya?, de su trabajo: velar por el territorio municipal; incluso por cualquier otro problema que acontece en otros municipios, el consejo del pueblo tiene que enlazarse para ayudar a los municipios... pero tenemos que estar organizados para levantar nuestra voz para defender lo nuestro, nuestra naturaleza porque somos los dueños. Aquí los trasnacionales nada tienen que venir a imponer leyes. Esa es la fuerza que tiene el

8 Gómez sostiene que «en el artículo 52 del Reglamento de la Ley de los Consejos de Desarrollo Urbano y Rural, se les puede definir: como instituciones de derecho público creadas para organizar las comunidades (aldeas), promoviendo la participación de sus habitantes en la búsqueda y promoción de su desarrollo» (Gómez, 2007:1).

9 En la página de la organización Servicios Jurídicos y Sociales (Serjus) de Guatemala se describe el objetivo de la conformación del Consejo Nacional de Autoridades Ancestrales de la siguiente manera: "La idea es de un sistema de gobierno de los pueblos mayas que tenga sus raíces en las comunidades, un sistema de consejos de autoridades ancestrales mayas, xincas y garífunas, como representación nacional e internacional de los Pueblos Mayas, que, además de tener sus raíces en las comunidades, las fortalezca en la lucha por defensa del territorio, de la administración de justicia comunitaria, de la espiritualidad, de las formas propias de organización, de la racionalidad económica maya» (Serjus, 2010: 8). 
pueblo, esa es la fuerza que tiene el Consejo de los Pueblos. Organizarnos a nivel municipio, agarrar también las autoridades comunitarias, incluso al alcalde municipal de lo que es nuestro municipio y a nivel departamental también podemos agarrarnos con el gobernador (Consejo del Pueblo Mam de Sibinal, comunicación personal, 6 de junio de 2017).

En efecto, existe por un lado una tensión constante entre el actuar de las autoridades gubernamentales y el reconocimiento al poder paralelo que significaría la incidencia del Consejo del Pueblo Mam en Sibinal. Con el apoyo y la orientación de los Consejos del Pueblo del departamento de San Marcos, en este municipio se realizaron, en 2010, los primeros intentos de conformar el Consejo del Pueblo, a pesar de la desconfianza de las autoridades oficiales de la municipalidad. Sin embargo, y por la insistencia y la movilización de las autoridades indígenas, en febrero de 2016 tuvo lugar un acto público durante el cual se inscribió en el libro de actas municipal el reconocimiento de las autoridades ancestrales maya mam organizadas en el Consejo del Pueblo Mam de Sibinal.

\section{Asociación civil Conciencia Cultural Mam}

En México, las comunidades mam asentadas actualmente en la región del Soconusco y en la Sierra Madre de Motozintla son resultado de procesos migratorios ${ }^{10}$ promovidos durante el gobierno de Porfirio Díaz, a finales del siglo XIX, que buscaban la colonización indígena de los terrenos de la serranía para contar con fuerza de trabajo disponible y barata para las fincas cafetaleras (Hernández, 2008). Durante este proceso, «el concepto de 'desarrollo' se convirtió para la población indígena del sureste mexicano en sinónimo de aculturación y contratación en las fincas cafetaleras» (Hernández, 2008: 53).

Durante los años treinta del siglo XX, el presidente Lázaro Cárdenas impulsó una reforma agraria, acompañada de una política nacionalista que se ha identificado como mexicanización forzada, y que tuvo particular importancia en la

10 La región del Soconusco, en particular las faldas del volcán Tacaná, es considerada parte del territorio mam habitado unos 2500 años antes de nuestra era (Monterroso, 2010; Quintana y Rosales, 2006); sin embargo, las comunidades actuales son el resultado de movimientos migratorios de origen guatemalteco hacia México para iniciar la conformación de pueblos y colonias (Peña, 2004). Existe un vacío de información sobre qué sucedió con los indígenas mam anteriores a la conformación de las comunidades que conocemos, pero distintos autores coinciden en la gran cantidad de procesos migratorios de la población mam (Robles, 2017). 
región fronteriza del Soconusco debido a que resultaba difícil para el gobierno mexicano distinguir la nacionalidad de la población mam que vivía en la sierra (Peña, 2004). Dicho proceso tuvo una repercusión negativa en la identidad cultural del pueblo mam, puesto que les prohibía de manera amenazante el uso de su lengua y su vestido (Fuentes, 2015; Peña, 2004).

En los años setenta del siglo XX, instituciones gubernamentales como el Instituto Nacional Indigenista (INI) modificaron su discurso de aculturación de las comunidades para integrarlas a la cultura nacional y «elevaron» la condición del indígena para volverlo copartícipe de la vida nacional (Sosa y Henríquez, 2012). Tata Nico, uno de los integrantes de la asociación Conciencia Cultural Mam, relata su experiencia en «el regreso» de la cultura mam en Unión Juárez:

Nosotros empezamos a perder nuestra lengua a la edad de doce años, ya nadie lo hablaba; y nos quedamos así, hablando solo español, pero verdaderamente empezamos... ora sí que a regresar a nuestra cultura por medio de un gobernador que fue... parece que fue Castillo Tielemans, ${ }^{11}$ él fue el que pidió que bajara un proyecto para los municipios que tenían que hablar, tener algunas personas hablantes de su propia lengua y ellos son los que se iban a encargar de solicitarlos (tata Nico, comunicación personal, 21 de mayo de 2017).

En el relato se comenta cómo se promovió -desde las estructuras del Estadoel retorno al uso de la lengua mam en sintonía con el replanteamiento del discurso oficial que reconocía a un México multicultural (Hernández, 2008).

...entonces fui nombrado como consejo indígena, ${ }^{12}$ yo y un señor que se llama Joaquín Verdugo y un tal Antonino Martínez. Pues nos nombraron así sin avisarnos a nosotros, sino que nos nombraron nada más a espaldas ¿qué decir? Sin consentimiento de nadie, pero a los ocho días de estar nombrado ni ellos nos notificaron, sino que nos Ilamaron a Unión Juárez con una orden judicial a los tres para que nos presentáramos (tata Nico, comunicación personal, 21 de mayo de 2017).

11 José Castillo Tielemans fue gobernador del estado de Chiapas de 1964 a 1970, durante la presidencia de Gustavo Díaz Ordaz en México.

12 Durante el gobierno de Luis Echeverría (1970-1976) se «buscó movilizar y dar voz a los indígenas, y para ello confió -el gobierno- a la Confederación Nacional Campesina la organización de los consejos indígenas y también la creación de organismos de funciones indefinidas que recibieron el nombre de consejos supremos» (Cordera y Ziccardi, 2000: 561). 
De manera externa a la comunidad -en una franca lógica heterónoma-, sin consulta de los propios involucrados, se nombró a los integrantes del consejo indígena para desempeñarse como instructores de la lengua mam en un contexto de desconfianza por parte de los indígenas -que hasta entonces se asumían como campesinos- hacia los nuevos planteamientos gubernamentales, motivada por los maltratos asociados a las políticas de mexicanización sufridos en los años precedentes. Sin embargo, hubo aceptación de estos nombramientos en virtud de que los campesinos poco a poco fueron descubriendo que al asumirse como parte del pueblo indígena mam tendrían acceso a becas, plazas como maestros mam hablantes, fondos para proyectos culturales y puestos en los Consejos Supremos (Hernández, 1994).

El INI y otras instituciones, como el Centro Coordinador Indigenista o el Departamento de Asuntos Indígenas, explican el surgimiento de distintos grupos que adoptaron, con una clara influencia gubernamental, el discurso del «rescate de la cultura» en la región Sierra y Soconusco de Chiapas ${ }^{13}$ y específicamente en Unión Juárez y Cacahoatán, donde se encuentra la asociación Conciencia Cultural Mam.

Ahora bien, no sería del todo correcto atribuir solo a las políticas gubernamentales el surgimiento de los grupos como si se tratara de actores pasivos y manipulables; en la actualidad existe una memoria, principalmente de los tatas, sobre la importancia que tuvo su labor independiente en el rescate de la cultura, sin recibir ninguna remuneración, con base en los modos de vida de las comunidades y en el recuerdo de los conocimientos de los abuelos mam. Así lo expresa tata Hermelindo, una de las figuras con mayor autoridad moral en la asociación:

Pues la idea de habernos conformado como Conciencia Cultural y como Raíces ${ }^{14}$ fue de que empezamos en primer lugar con la cuestión de la lengua que ya casi nadie lo quería hablar y había mucha discriminación en contra de la lengua. Pero nace la idea de ponernos de acuerdo con otro compañerito para ver si la gente gustarían nuevamente volver a recordar la lengua y empezamos a hacer algunas reuniones y aun-

13 Para profundizar en el proceso de consolidación de las instituciones gubernamentales y en el discurso del rescate cultural se recomienda el texto La otra frontera. Identidades múltiples en el Chiapas Poscolonial, donde Hernández Castillo (2001) describe el replanteamiento de las políticas de integración nacional de los pueblos indígenas al Estado mexicano, así como la modificación del discurso oficial hacia el reconocimiento de un México multicultural durante los años setenta del siglo XX.

${ }_{14}$ Se refiere a Raíces de Nuestra Cultura Mam, grupo surgido en la década de los noventa del siglo XX, al cual pertenece, y que su vez forma parte de la asociación civil Conciencia Cultural Mam. 
que estuvo un poco difícil porque tuvimos que movernos a través de nuestros propios recursos, destinamos tiempo, visitamos algunas comunidades, partes donde fuimos aceptados y partes donde no (...) Pero a través de los días que fuimos pasando ya nos dimos cuenta que para hablar de la cultura de los pueblos originarios no solamente era hablar o enseñar a hablar o escribir sino que surge que empezamos a pensar que era necesario volver a los conocimientos de los abuelos (tata Nico, comunicación personal, 24 de mayo de 2017).

El referirse a los conocimientos de los abuelos como elemento del rescate cultural adquiere relevancia en el desenvolvimiento de la Asociación Civil Conciencia Cultural Mam, debido a que se conduce la mirada a distintas prácticas y modos de vida con los que, consideran, se identifican como pueblo. En las actividades en las que fue posible participar durante el trabajo de campo se observó un sobresaliente respeto hacia la palabra de los abuelos, así como a la autoridad moral que les significa.

Entre las organizaciones que se consolidaron durante los años noventa cabe mencionar el Consejo Supremo Mam, el Consejo Regional Indígena Mam del Soconusco, Promotores Culturales de la Lengua Mam, Raíces de Nuestra Cultura Mam y Conciencia Cultural Mam, antecesores de la asociación civil seleccionada para este análisis. Actualmente, aunque con ciertas dificultades, solo sobreviven dos: Consejo Regional Indígena Mam del Soconusco y Raíces de Nuestra Cultura Mam, que representaron a los más visibles en Unión Juárez y Cacahoatán. Algunos de los integrantes de la Asociación Civil Conciencia Cultural Mam han pertenecido a los grupos mencionados y con base en su experiencia organizativa han impulsado la consolidación de la asociación desde mediados de 2015.

\section{Mirada hacia dos rumbos}

Para el acercamiento a las organizaciones descritas se tomó en cuenta el planteamiento de Gustavo Esteva, quien ubica dos grandes impulsos contrapuestos en las luchas por la autonomía indígena latinoamericana: 1) el que considera necesaria la toma del poder y la afiliación a la democracia formal para regular y controlar la autonomía dentro del régimen estatal existente y 2) el de quienes reafirman la autonomía en sus propios espacios e intentan generar un cambio social radical reorganizando la sociedad desde abajo (Esteva, 2016). Esta clasificación permite pensar en las diferencias cualitativas de las autonomías indígenas 
y ubicar una tendencia de los sujetos de estudio para el análisis de la dicotomía entre autonomía y heteronomía.

En el primer impulso descrito por Esteva se observan características del ejercicio del poder gubernamental que se acomoda como parte de la heteronomía sobre los pueblos indígenas. En el segundo, se percibe un sentido de transformación social desde los espacios locales y por ello se retoma el concepto de autonomía desde la perspectiva de la comunalidad, la cual presenta una base cultural para su planteamiento, además de que se sustenta en cuatro aspectos básicos o condiciones propicias para ejercer la autonomía indígena: territorio, poder, trabajo y disfrutes comunales (Maldonado, 2002). En palabras de Benjamín Maldonado:

Es precisamente la comunalidad la que constituye y es capaz de crear (recrear) las condiciones necesarias para la autonomía: la reciprocidad basada en el principio de la ayuda mutua, el poder en manos del colectivo constituido en asamblea, la voluntad de servir gratuitamente durante años a la comunidad en diversos cargos a pesar de ser onerosos, la defensa de un territorio histórica y culturalmente propio, son elementos suficientes para un régimen de autonomía en condiciones propicias y esas condiciones son las que confisca el Estado: dependencia administrativa, economía de mercado, no decisión sobre el territorio comunitario y un sistema de vinculación con los municipios basado en el despojo de poder a través de caciques, delegados de gobierno y diputados (Maldonado, 2002: 77).

De manera que, para conocer si existe una noción de autonomía en los discursos y las prácticas de los grupos Conciencia Cultural Mam y el Consejo del Pueblo Mam de Sibinal fue necesario reflexionar sobre sus objetivos y aspiraciones, ya que esto hace posible conocer la proyección que tienen hacia el futuro y con base en ello pensarlos desde su subjetividad política y su capacidad de agencia. Por otra parte, la relación con los poderes gubernamentales en cuestión permite conocer cómo se han establecido algunos mecanismos de dependencia organizativa para su funcionamiento.

En primera instancia, los objetivos planteados por el Consejo del Pueblo Mam de Sibinal giran en torno a una actividad central: velar por el territorio en que viven, defenderlo ante cualquier intromisión externa, en referencia principalmente a la extracción minera y la operación de plantas hidroeléctricas que se han establecido en distintos lugares del departamento de San Marcos: «Y entonces viene la idea que cuál era el plan del consejo de los pueblos, de su trabajo: velar 
por el territorio municipal; incluso por cualquier otro problema que acontece en otros municipios, el consejo del pueblo tiene que enlazarse para ayudar a los municipios» (Consejo del Pueblo Mam de Sibinal, comunicación personal, 6 de junio de 2017).

La defensa del territorio es un objetivo compartido con otros consejos mam de los municipios del departamento de San Marcos, con los cuales están articulados y vinculados a nivel organizativo (Yagenova y García, 2009). Este vínculo explica que en su discurso se mencione la defensa del territorio, aunque en Sibinal no exista actualmente la intromisión de alguna empresa minera o la operación de algún proyecto hidroeléctrico. ${ }^{15}$ Sin embargo, expresa, por un lado, que se reconocen como integrantes de un territorio común, y por otro, que asumen la incidencia de la economía extractiva trasnacional como una amenaza externa y latente que puede afectar sus comunidades.

Además, se evidencia que la ayuda mutua y la solidaridad forman parte de su práctica organizativa y así ha quedado de manifiesto en distintas actividades, como el acto de bienvenida a los presos políticos del municipio de San Pablo ${ }^{16}$ en abril de 2017, que al mismo tiempo resultó en una denuncia hacia el proceso penal en contra de las autoridades mayas y comunitarias. En esta misma actividad se exigió una vez más el respeto a las autoridades indígenas y a su derecho de ser consultados ${ }^{17}$ como pueblos ante cualquier asunto que pueda afectarlos en su territorio (Asamblea Te Txe Chman, 2017).

En una de las entrevistas se expresa el sentir de un integrante del consejo en torno a sus aspiraciones y horizontes de lucha política, así como su percepción hacia los poderes gubernamentales, en una actitud de desconfianza hacia las

15 Aunque haya un antecedente en una nota periodística en que incluyen el municipio de Sibinal como parte de los municipios del departamento de San Marcos en que existen algunas concesiones para la extracción minera (Vogt, 2005), actualmente no hay información reconocida por el Ministerio de Energía y Minas del gobierno de Guatemala en este sentido (Gobierno de la República de Guatemala, s./f).

16 En 2008 el municipio de San Pablo, en el departamento de San Marcos, inicia el proceso de resistencia comunitaria ante la inconformidad por el ingreso de maquinaria perteneciente a la empresa Hidrosalá que forma parte del grupo Fabrigas y que tuvo la intención de construir un proyecto hidroeléctrico en la finca Argentina en ese municipio. En marzo de 2014 un camión de la empresa Hidrosalá se incendió, pero el incidente no fue esclarecido. Ante estos acontecimientos fueron detenidos diez pobladores de San Pablo bajo los cargos de asociación ilícita, plagio, detenciones ilegales, entre otros. En abril de 2017 se realizó un acto de bienvenida a seis de los diez presos políticos de San Pablo, uno de ellos reconocido como autoridad maya mam de ese municipio.

17 El derecho de consulta asumido por los pueblos indígenas tiene sustento jurídico a nivel internacional en el Convenio 169 de la Organización Internacional del Trabajo (OIT) y por ello la enarbolan como un derecho base para sus propias formas de organización. 
autoridades legalmente reconocidas por el Estado, así como hacia el sistema de partidos políticos convencionales:

Y el resultado que queremos obtener es ya no creer en partidos políticos que solo nos vienen a engañar, a imponer sus leyes, pero ya al estar ahí no se cumple lo que ofrecen sino son otras las consecuencias que se reciben. Ya sus reglas... las autoridades pues siguen siendo autoridades encima del pueblo, de los pueblos. Y lo que queremos ahora es de que vayamos teniendo la fuerza de todo el pueblo para que nosotros algún día podamos gobernar nuestro mismo pueblo, porque si logramos poner nuestro propio gobierno creo que ya va a ser diferente porque las necesidades ya se conocen. No hay de que se va el dinero del país; pues no se mira, no regresa, no se ve, aunque aporta pero no hay. En cambio, si llega una nuestra persona que va a nuestro favor pues pueda que cambie nuestro país, nuestro desarrollo es lo que deseamos (Consejo del Pueblo Mam de Sibinal, comunicación personal, 6 de junio de 2017).

Este extracto de la entrevista muestra, por un lado, la aspiración de autogobierno como una visión a futuro que puede ser considerada medular en la construcción de autonomía y, por el otro, el reconocimiento tácito a un régimen heterónomo existente. Sin embargo, el hecho de que refieran un cambio en el país a partir de la llegada al poder de una persona que va a nuestro favor también puede percibirse como una confianza en las estructuras convencionales y jerárquicas del poder gubernamental. Además, utilizan el concepto de desarrollo para expresar su aspiración general, lo que puede reflejar la influencia del discurso de las instituciones municipales oficiales con respecto a los planes de desarrollo.

En cuanto a los objetivos expuestos por los integrantes de la Asociación Civil Conciencia Cultural Mam, hay menos homogeneidad en la visión de sus integrantes y por lo menos se han ubicado dos objetivos prioritarios: 1) el rescate de la cultura mam, principalmente a través de la enseñanza de la lengua; y 2) el acceso a recursos económicos a través de apoyos institucionales y a programas sociales y proyectos productivos otorgados por instancias gubernamentales. Así lo expresa el presidente de la asociación civil: «uno de los objetivos de la A.C. es precisamente para consolidarnos más y pues más que nada la visión de los compañeros era y sigue siendo para aterrizar algún proyecto, pues, aterrizar un proyecto» (Conciencia Cultural Mam, comunicación personal, 1 de junio de 2017).

«Aterrizar un proyecto» se entiende como sinónimo de obtención de recursos económicos gubernamentales. En este sentido, para los integrantes del grupo, 
la relación que existe entre los dos objetivos mencionados es directa o, dicho de otro modo, perciben la obtención de recursos económicos como un elemento necesario para promover el rescate cultural por medio de la enseñanza de la lengua o para realizar actividades culturales en espacios públicos. Por ello, ante este escenario, no han fomentado actividades que les permitan obtener recursos colectivos por cuenta propia y siguen visualizando las instituciones gubernamentales como las principales fuentes que pueden proveerlos de tan anhelados recursos, lo cual los posiciona, por un lado, en una situación de dependencia y, por otro, afirma el carácter asistencialista y paternalista del Estado, herencia del indigenismo institucionalizado que concluyó a inicios del siglo XXI. ${ }^{18}$

Sin embargo, ¿será correcto pensar las aspiraciones de la asociación únicamente desde el lugar de la heteronomía? Si bien es cierto que está entre sus objetivos la obtención de apoyos gubernamentales para realizar sus actividades, la determinación de rescatar la cultura mam con la cual se identifican los ha conducido por nuevos caminos organizacionales. El rescate cultural, si bien fue promovido inicialmente por las instituciones gubernamentales en la década de los setenta (Hernandez, 2001), en la actualidad ha sido asimilado y resignificado al grado de que es posible pensarlo como un elemento sobre el que se posicionan como indígenas mam y que los fortalece a pesar de haber sido afectados por factores tan remotos como la invasión española, los procesos de colonización, o más próximos, como las políticas de mexicanización que les prohibían hablar la lengua mam y utilizar su vestimenta tradicional.

Cuando hablan de rescate cultural hacen referencia a costumbres, tradiciones, formas de alimentación, de trabajo con la tierra, saberes, y modos de vida que los identifican como un pueblo cuyas prácticas sobreviven a pesar del intento de exterminio al que han sido sometidos sistemáticamente. Con respecto al trabajo con la tierra, tata Hermelindo dijo:

Bueno, si planteáramos esto a las dependencias de gobierno: no a la minería, no a los fertilizantes, no al insecticida, no al fungicida, pues ellos no estarían de acuerdo por-

18 Sámano afirma que «La política del indigenismo institucionalizado pretendió la integración del indígena a través de la acción del Estado, para lograr hacer justicia social atendiendo a este sector de la población que se había mantenido en el olvido; sin embargo, el indigenismo derivó en asistencialismo y paternalismo. Se quiso integrar a los indígenas a la nación, pero al mismo tiempo algunos antropólogos querían que conservaran sus costumbres ancestrales; en esta política ambivalente del indigenismo mexicano, si se desaparecía o no a los indígenas por medio de una política gubernamental, era una de las disyuntivas a las que se enfrentaron los indigenistas» (Sámano, 2004: 16). 
que de esta manera nosotros estaríamos quitando un negocio a ellos y siempre van a insistir en que el fertilizante es esto, en que el fertilizante es lo otro. Entonces es un trabajo y es una lucha que tenemos de mantenernos en este estado como pueblo originario y seguir inculcando en la gente que mantengamos nuestras costumbres, nuestra manera de trabajar como nos lo enseñaron nuestros abuelos, de mantener el suelo así, a lo natural (Conciencia Cultural Mam, comunicación personal, 25 de abril de 2017).

Una posibilidad que se ha visualizado durante la investigación es que la búsqueda de rescate cultural al que se ha hecho referencia exige mirar la manera en que las comunidades y organizaciones de Guatemala practican la espiritualidad y todo el abanico de costumbres y saberes que las ubican como parte de la cultura mam, en cuyos espacios de encuentro están comenzando a tener resonancia el elemento organizativo y las figuras de autoridad indígena. Lo anterior refuerza su identidad como pueblo y los hermana con diferentes actores del movimiento indígena de Guatemala, con quienes han tejido redes de solidaridad y de mutuo aprendizaje con miras al fortalecimiento de su cultura.

\section{Reflexiones finales}

Con lo expuesto hasta ahora es posible afirmar que la noción de autonomía no está presente de manera explícita en el discurso de ambos grupos, aunque sus aspiraciones muestran algunos rasgos que pueden ser considerados parte de las condiciones propicias para que un régimen autonómico pueda desenvolverse, particularmente en el caso de Guatemala. El hecho de que el concepto de autonomía no sea una prioridad explícita en su discurso, no impide pensar en las potencialidades de sus aspiraciones y en sus figuras organizativas, las legitimidades, búsquedas y acciones encaminadas a resignificar un territorio que ambas organizaciones asumen como propio.

La relación histórica que sostienen los pueblos indígenas con los Estados nacionales es un elemento que permite comprender el desenvolvimiento y las aspiraciones de las organizaciones indígenas en general, y de las organizaciones mam en particular. En México ha existido una relación de dependencia a nivel organizativo y político que se verifica en el caso de estudio y que puede ubicarse desde la consolidación de una forma estatal que se funda en el despojo y la tutela, donde queda asentado el control heterónomo de los pueblos indígenas, y que tiende a inhibir, deformar o anular las capacidades políticas comunitarias 
y autónomas (Linsalata, 2016). En Guatemala, los procesos de conformación del Estado han sido caracterizados por el racismo y la violencia ejercidos de manera sistemática sobre los pueblos indígenas (Cojtí Cuxil, 2005). Las particularidades de sus procesos de negociación y conflicto con el Estado, en ambos países, se materializan en iniciativas con distintas expresiones y alcances, bajo la influencia también de los vínculos y referentes del movimiento social organizado.

Para el Consejo del Pueblo Mam de Sibinal, la reivindicación de la identidad indígena adquiere relevancia en cuanto a su función política, ya que permite entender las relaciones de poder que de alguna u otra manera los limita en la búsqueda de sus aspiraciones, y es a partir de esta limitante que se afirman como autoridades indígenas para recuperar parte de ese poder negado. Al respecto, Korsbaek afirma que «la problemática de la identidad étnica es en un primer plano un problema político, y solamente en un segundo plano es un problema cultural. Se postula aquí que un grupo étnico posee una identidad que se define así: un proyecto político que, con base en un acervo cultural, pretende convertirse en una realidad social y de esta manera forjar el proceso histórico» (Korsbaek y Sámano, 2007: 214).

Por el contrario, en la asociación Conciencia Cultural Mam se asume la identidad indígena desde el ámbito cultural, sin un claro horizonte de organización política a nivel comunitario. Sin embargo, esto puede modificarse debido a la disponibilidad que existe para conocer y replicar las formas de organización que están consolidándose en los municipios vecinos de Guatemala, convirtiéndose los consejos del pueblo en su principal referente político. Además, es posible afirmar que actualmente se vive una continuidad del proceso de hermanamiento entre los indígenas mam en ambos países, donde la identidad cultural juega un papel relevante para, eventualmente, afirmarse como sujetos políticos con capacidad de agencia.

En el proceso de inclusión e incidencia política desde la subjetividad, el Consejo del Pueblo de Sibinal sostiene una lucha por el reconocimiento de las figuras de autoridad indígena ancestral y busca influir en la toma de decisiones ante diversas situaciones de carácter comunitario, con un discurso que privilegia la defensa del territorio. Por otro lado, Conciencia Cultural Mam se ubica como una asociación cercana a las instituciones del Estado mexicano, en colaboración con los gobiernos municipales, que adecua su discurso y sus prácticas a intereses de carácter cultural en actividades públicas que, la mayoría de las veces, adquiere un sentido de espectáculo y entretenimiento que favorece a los poderes 
locales y la promoción del turismo regional, donde la motivación por obtener recursos es una aspiración latente.

En el consejo de Guatemala existen prácticas más arraigadas en la cotidianidad que pueden tomarse como parte del conocimiento cultural: la politización, la defensa territorial, la rememoración constante, el sistema de cargos, la invocación de agradecimiento al inicio y término de alguna reunión o actividad, la rameada con la hoja del maíz; sin embargo, la identidad explícita no es tan recurrente en su discurso. En contraste, el grupo de México habla constantemente de su identidad indígena y del rescate cultural, pero en la vida cotidiana se observa una búsqueda y asimilación de la cultura occidental y un acatamiento al régimen legal institucionalizado.

Ambos grupos muestran interés en recibir capacitaciones y asesorías en el ámbito jurídico para dar mayor sustento a su legitimidad como autoridades indígenas y para adquirir fortaleza a nivel organizativo, además de que están al pendiente de su legitimidad como autoridades ancestrales ante las comunidades a las que pertenecen con base en distintos documentos institucionales, apegados a las normas jurídicas sostenidas por los Estados nacionales en cuestión, lo cual puede mostrar una relación de subordinación, pero también de exigencia y afirmación de sus derechos como comunidades indígenas.

En suma, entre ambas organizaciones existe una diferencia central que muestra el grado de ejercicio de la autonomía indígena relativa en cada caso: la cuestión del poder en su dimensión política. El hecho de que el Consejo del Pueblo Mam asuma el ejercicio de la autoridad ancestral y la defensa del territorio como ejes de su desenvolvimiento organizativo refleja una afirmación desde su subjetividad tendiente a la construcción de un gobierno propio reconociendo el poder de la asamblea comunitaria en un espacio que posibilita su reproducción cultural, social, histórica y económica. El hecho de que la asociación civil Conciencia Cultural Mam considere necesaria la obtención de recursos otorgados por instituciones gubernamentales para el rescate cultural y la enseñanza de la lengua, sin una aspiración de autoridad en el sentido político, refleja la asimilación del poder externo, heterónomo, que delega el poder político organizativo a instancias ajenas a las comunidades y que vulnera la toma de decisiones en sus espacios de reproducción social, cultural y económica. 


\section{Agradecimientos}

Agradecemos la colaboración de Valentina Valle Baroz en la revisión del texto original. A las familias González, Verduo y Mejía por la confianza para abrirnos las puertas de sus hogares y posibilitar el acercamiento al territorio del pueblo mam en la frontera binacional.

\section{Fuentes de consulta}

Alonso, Jorge y Rafael Sandoval Álvarez, 2015, «Sujeto social y antropología. Despliegue de subjetividad como realidad y conocimiento», en Jorge Alonso y Rafael Sandoval Álvarez (coords.), Pensamiento crítico, sujeto y autonomía, México, Centro de Investigaciones y Estudios Superiores en Antropología Social.

Asamblea Te Txe Chman, 2017, Declaración del Encuentro de Comunidades y Pueblos por la Vida, la Libertad y la Defensa de Nuestros Ríos y Territorios, comunicado inédito, San Marcos, Guatemala.

Bartra, Armando, 1995, "Origen y claves del sistema finquero del Soconusco», en Neus Espresate (ed.), Chiapas 1, Instituto de Investigaciones Económicas-Universidad Nacional Autónoma de México/ Era, México, pp. 29-52.

Bengoa, José, 2000, La emergencia indígena en América Latina, México, Fondo de Cultura Económica.

Bonvillani, Andrea, 2014, "Saberes apasionados: horizontes de construcción de conocimiento de las subjetividade(s) política(s)», en Claudia Piedrahita Echandía, Álvaro Díaz Gómez y Pablo Vommaro (comps.), Acercamientos metodológicos a la subjetivación política: debates latinoamericanos, Colombia, Universidad Distrital Francisco José de Caldas, Consejo Latinoamericano de Ciencias Sociales, pp. 83-100.

Cojtí Cuxil, Waqi' Q'anil Demetrio, 2005, El racismo contra los pueblos indígenas de Guatemala, Guatemala, Consejo Nacional de Educación Maya.

Colino, César, 2009, «El método comparativo», en Diccionario Crítico de Ciencias Sociales. Terminología Científico-Social, Madrid-México, Plaza y Valdés.

CPO Consejo del Pueblo Maya, 2014, «Proyecto político. Un nuevo Estado para Guatemala: democracia plurinacional y gobiernos autónomos de los pueblos indígenas», Quetzaltenango, Guatemala, Consejo del Pueblo Maya (mns).

Cordera, Rolando y Ziccardi Alicia (coords.), 2000, Las políticas sociales de México al fin del milenio: descentralización, diseño y gestión, México, UNAM.

Declaración de pueblos originarios, Declaración de pueblos originarios, Tapachula, Chiapas México y Guatemala, en el encuentro de sabidurías ancestrales para la defensa de la vida, la madre tierra y sus bienes naturales, 2016, Tapachula, Chiapas (mns), El Colegio de la Frontera Sur, en <http://www.ecosur.mx/wp-content/uploads/2016/02/Declaracio\%CC\%81n-de-pueblos-originarios-TapachulaChiapas-Me\%CC\%81xico-y-Guatemala-en-el-Encuentro-de-Sabiduri\%CC\%81as- 
Ancestrales-para-la-defensa-de-la-vida-la-Madre-Tierra-y-sus-bienes-naturales. pdf $>$.

Esteva, Gustavo, 2016, «La hora de la autonomía», en Luciana García Guerreiro y Pavel López Flores (coords.), Pueblos originarios en lucha por las autonomías: experiencias y desafíos en América Latina, Buenos Aires, CLACSO/Editorial El Colectivo, pp. 29-57.

Franco, Yago, 2017, «Heteronomía», Magma, en <http://www.magma-net.com.ar/glosario.htm>.

Fuentes Malo, Sinue Hammed, 2015, Las memorias del proceso de mexicanización del pueblo mam en el Soconusco, Chiapas, tesis de maestría inédita, Tapachula de Córdova y Ordóñez, Chiapas, México, El Colegio de la Frontera Sur.

García Guerreiro, Luciana y Pavel López Flores (coords.), 2016, Pueblos originarios en lucha por las autonomías: experiencias y desafíos en América Latina, Buenos Aires, CLACSO/Editorial El Colectivo.

Gatica Polco, Daniel, 2015, «El territorio de los pueblos originarios frente a la lógica del neoliberalismo», Revista Mexicana de Ciencias Agrícolas, 1, pp. 191-197.

Gobierno de la República de Guatemala, s./f, «Derechos mineros otorgados por departamento», en <http://www.mem.gob.gt/mineria/catastro-minero/ derechos-mineros-otorgados-por-depto/>.

Gómez Chacón, Mario Rodrigo, 2007, Análisis jurídico de los consejos comunitarios de desarrollo como un mecanismo para la descentralización administrativa del Estado guatemalteco, tesis de licenciatura inédita, Guatemala, Universidad de San Carlos.

González, Miguel, Araceli Burguete Cal y Mayor y Pablo Ortiz (coords.), 2010, La autonomía a debate: autogobierno indígena y estado plurinacional en América Latina, Guadalajara, México, Editorial Universitaria.

Hernández Castillo, Rosalva Aída, 1994, «ldentidades colectivas en los márgenes de la nación: etnicidad y cambio religioso entre los mames de Chiapas», Nueva Antropología. Revista de Ciencias Sociales, X(45), pp. 83-105.

Hernández Castillo, Rosalva Aída, 2001, La otra frontera. Identidades múltiples en el Chiapas poscolonial, México, CIESAS.

Hernández Castillo, Rosalva Aída, 2008, Procesos contemporáneos de conformación de identidades indígenas en la frontera sur de Chiapas, Cuadernos de Investigación, México, Comisión Nacional para el Desarrollo de los Pueblos Indígenas, pp. 53-77.

Hernández Castillo, Rosalva Aída, Sarela Paz y María Teresa Sierra (coords.), 2004, El Estado y los indígenas en tiempos del PAN: neoindigenismo, legalidad e identidad, México, CIESAS.

Jerónimo Sánchez, Eutiquio et al., 2007, Tlahtolnechikolli Diccionario Nawatl Moderno I Español de la Sierra de Zongolica, Veracruz, Xalapa, Xochitlahtolli.

Jiménez Sánchez, Odilio, 2008, Los caminos de la resistencia: comunidad, política e historia maya en Guatemala, tesis de doctorado inédita, Austin, Universidad de Texas.

Korsbaek, Leif y Miguel Ángel Sámano Rentería, 2007, «El indigenismo en México: antecedentes y actualidad», Ra Ximhai, 3(1), pp. 195-224.

Lenkersdorf, Gudrun, 2016, Concejos y caciques. Resistencia organizada en el mundo maya, México, Pensaré Cartoneras. 
León Ardiano, Denny Rafael de (2016), El consejo maya mam del departamento de San Marcos como sistema de organización propia y autoridad indígena para la promoción de los derechos de los pueblos indígenas ante la institucionalidad del estado, tesis de licenciatura inédita, Guatemala, Universidad de San Carlos.

León, Quimy de y Santiago Bastos, 2015, "Guatemala: construyendo el desarrollo propio en un neoliberalismo de posguerra», Revista Pueblos y fronteras digital, 10(19), pp. 52-79.

Limón Aguirre, Fernando, 2010, Conocimiento cultural y existencia entre los chuj, México, Comisión Nacional para el Desarrollo de los Pueblos Indígenas.

Linsalata, Lucia, 2016, Lo comunitario-popular en México: desafíos, tensiones y posibilidades, México, Benemérita Universidad Autónoma de Puebla.

López Bárcenas, Francisco, 2006, «Autonomía y derechos indígenas en México», Cuadernos Deusto de Derechos Humanos, 39, Bilbao, Universidad de Deusto.

López y Rivas, Gilberto, 2004, Autonomías, democracia o contrainsurgencia, México, Ediciones Era.

Maldonado Alvarado, Benjamín, 2002, Autonomía y comunalidad india. Enfoques y propuestas desde Oaxaca, Oaxaca, Centro INAH, Secretaría de Asuntos Indígenas del Gobierno del Estado de Oaxaca, en <http://www.acratie.eu/FTPUTOP/MEXMALDONADO-Autonomiaycomunalidad.pdf $>$.

Medina Hernández, Andrés, 1973, «Notas etnográficas sobre los mames de Chiapas», Anales de Antropología, en <http://www.revistas.unam.mx/index.php/antropologia/article/view/23286/pdf_720> (consulta: 04/11/2018).

Monterroso, Juan José, 2010, «Q’ chman, aye' q' chmam, nuestros abuelos-as y nietos-as: guardianes y señores del tiempo y de la tierra», Génesis del Pueblo Maya (archivo electrónico), en <https://docplayer.es/23284266-Q-chman-aye-q-chmam-nuestrosabuelos-as-y-nietos-as-guardianes-y-senores-del-tiempo-y-de-la-tierra.html>.

Peña Piña, Joaquín, 2004, Migración laboral de las mujeres y estrategias de reproducción social en una comunidad indígena Mam de la Sierra Madre de Chiapas, tesis doctoral inédita, México, El Colegio de la Frontera Sur.

Peña Piña, Joaquín y Andrés Fábregas Puig, 2015, «Frontera, procesos migratorios y autonómicos en la conformación territorial mam: cambios y perspectivas», LiminaR, 13(2), pp. 62-83.

Pérez Liñán, Aníbal, 2008, «El método comparativo: fundamentos y desarrollos recientes», documento de trabajo 1, Política comparada, pp. 1-29, en <https://burguete45.files.wordpress.com/2013/01/mc3a9todo-comparativo.pdf $>$.

Quintana Hernández, Francisca y Cecilio Luis Rosales, 2006, Mames de Chiapas, México, Comisión Nacional para el Desarrollo de los Pueblos Indígenas.

Reyes Ramos, María Eugenia, 2002, «Situación agraria y conflictos sociales en municipios indígenas de Chiapas», archivo electrónico, en <http://www.nacionmulticultural. unam.mx/edespich/images/diagnostico_y_perspectivas/Economia_sociedad_y_ desarrollo/Situacion_agraria_y_conflictos_sociales/ensayo_situacion_agraria.pdf $>$.

Reyes Ramos, María Eugenia y Álvaro Fernando López Lara, 1994, «Historia de la política agraria en Chiapas: el conflicto por la tierra», El Cotidiano, 62, mayo-junio, en <www. elcotidianoenlinea.com.mx/pdf/6203.doc>. 
Rivera Almaguer, Raquel Xochiquetzal, 2005, Autonomía indígena en México, tesis de maestría inédita, México, Universidad Iberoamericana, en <http://www.bib.uia.mx/ tesis/pdf/014599/014599.pdf>.

Robles Santana, María Aránzazu, 2017, «Las otras fronteras. Migración de mujeres indígenas Mam al Soconusco, México», Astrolabio, 19, pp. 231-245.

Sámano Rentería, Miguel Ángel, 2004, «El indigenismo institucionalizado en México (1936-2000): un análisis», en José Emilio Ordóñez (coord.), La construcción del Estado nacional: democracia, justicia, paz y estado de derecho. XII Jornadas Lascasianas, México, UNAM, pp. 141-158, en <http://biblio.juridicas.unam.mx/libros/3/1333/10. pdf>.

Sandoval Álvarez, Rafael, 2016, Formas de hacer metodología en la investigación, Guadalajara, Grietas.

Sartori, Giovanni y Leonardo Morlino, 1999, La comparación en las ciencias sociales, España, Alianza Editorial.

Serjus, 2010, Sentido y objetivo del trabajo con las Comunidades y Autoridades Ancestrales de los Pueblos Mayas, en <http://www.serjus.org.gt/pagina/node/120>.

Smeke, Yemy, 2000, «La resistencia: forma de vida de las comunidades indígenas», El Cotidiano, 16(99), pp. 92-102.

Sosa Suárez, Margarita y Cristina Henríquez Bremer, 2012, Instituto Nacional Indigenista / Comisión Nacional para el Desarrollo de los Pueblos Indígenas 1948 - 2012, México, CDI.

Spenser, Daniela, 1988, El Partido Socialista Chiapaneco. Rescate y reconstrucción de su historia, México, CIESAS.

Tapia, Marcela Aurora, 2017, «Las fronteras, la movilidad y lo transfronterizo: reflexiones para un debate», Estudios Fronterizos, 18(37), pp. 61-80.

Tarrío García, María y Luciano Concheiro Bórquez, 2006, «Chiapas: los cambios en la tenencia de la tierra», Argumentos, 19(51), pp. 31-71.

Torre González, Abelardo de la, 2015, La negociación del tratado de límites entre México y Guatemala -1853 a 1902-, Tapachula, s./e.

Velasco Cruz, Saúl, 2003, El movimiento indígena y la autonomía en México, México, UNAM.

Vogt Vehige, Daniel Joseph, 2005, «Intereses trasnacionales sobre las minas de Guatemala», Terrelibere, en <https://www.terrelibere.org/574-intereses-transnacionales-sobre-lasminas-de-guatemala/>.

Yagenova, Simona y Rocío García, 2009, «Guatemala: el pueblo de Sipakapa versus la empresa minera Goldcorp», Osal, 10(25), pp. 65-77.

\section{Cómo citar este artículo:}

Toledo Pineda, Miguel Ángel Cristhian y Enrique Coraza de los Santos, 2019, «Los mam de México y Guatemala: un pueblo binacional entre la autonomía y la heteronomía», Revista Pueblos y fronteras digital, volumen 14, e-369. https://doi.org/10.22201/cimsur.18704115e.2019.v14.369 